\title{
Efecto de la fertilización fosforada sobre el contenido de cadmio en cuatro suelos de Chile ${ }^{(1)}$
}

\author{
Claudia Bonomelli(2), Carlos Bonilla(2) y Adriana Valenzuela ${ }^{(2)}$
}

\begin{abstract}
Resumen - Los fertilizantes fosforados pueden contener cadmio (Cd) y al utilizarlos pueden entrar en las cadenas tróficas. El objetivo de este estudio fue evaluar el efecto de la fertilización fosforada sobre la concentración de Cd disponible en cuatro tipos de suelos de Chile, que pertenecían a los ordenes Inceptisol, Alfisol, Ultisol y Andisol. Los dos tratamientos usados fueron el testigo sin fertilización y la aplicación de una dosis de corrección con un fertilizante comercial, superfosfato triple (SFT), que tenía una concentración de 53,2 mg de Cd por kg de fertilizante. La dosis de fertilizante aplicada fue la necesaria para alcanzar un nivel de $30 \mathrm{mg} \mathrm{kg}^{-1}$ de P-Olsen. Los suelos se incubaron en estufa durante 90 días, a $25^{\circ} \mathrm{C}$ y humedad de capacidad de campo. El diseño experimental fue completamente al azar, con tres repeticiones para cada suelo y donde la unidad experimental fue un contenedor con $250 \mathrm{~g}$ de suelo seco. Se midió Cd disponible a los 1, 2, 7, 14, 21, 36, 49, 63, 77 y 90 días después de la incubación. La aplicación de $\mathrm{P}$ en dosis agronómica, utilizando fertilizante con alto contenido de Cd, tuvo un efecto estadísticamente significativo sobre la concentración de Cd disponible en los suelos estudiados, sin embargo, no hubo efecto del tiempo de incubación en la disponibilidad de Cadmio.
\end{abstract}

Términos para índece: metales pesados, sorción del suelo, contaminación, niveles tróficos.

\section{Effect of phosphate fertilizer on the soil cadmium content in four types of Chilean soils}

\begin{abstract}
Phosphate fertilizers may contain cadmiun (Cd) which may become part of the trophic chains. The objective of this study was to evaluate the effect of phosphate fertilizer on available Cd content in four Chilean soil types, belonging to orders Alfisol, Inceptisol, Ultisol and Andisol. Treatments consisted of control without $\mathrm{P}$ fertilizer and $\mathrm{P}$ application with commercial triple superphosphate containing $53.2 \mathrm{mg} \mathrm{Cd}$ per $\mathrm{kg}$ of fertilizer. The dose of phosphate fertilizer applied was enough to reach the level of $30 \mathrm{mg} \mathrm{kg}^{-1} \mathrm{P}$-Olsen. The soils were incubated up to 90 days, at $25^{\circ} \mathrm{C}$ and field capacity. The experimental design was completely randomized with three replications, and the experimental unit was a container with $250 \mathrm{~g}$ of soil (dry matter). Available Cd was measured at 1, 2, 7, 14, 21, 36, 49, 63, 77, and 90 days after incubation. Application of $\mathrm{P}$ at agronomic rate using fertilizer with a high Cd content produced significant effects on available Cd content in the soil types studied, however, incubation time period did not present such effect.

Index terms: heavy metals, soil sorption, contamination, trophic levels.
\end{abstract}

\section{Introducción}

En los últimos años, la presencia de Cd en los suelos y el riesgo de ingreso de este elemento a la cadena alimenticia, ha generado mundialmente una

\footnotetext{
(1) Aceptado para publicación en 17 de julio de 2003.

(2) Pontificia Universidad Católica de Chile, Facultad de Agronomía e Ingeniería Forestal, Casilla 306, Correo 22 Santiago, Chile. E-mail: cbonomel@puc.cl, cbonilla@puc.cl, aavalenz@puc.cl
}

preocupación creciente, debido al efecto tóxico de este elemento en humanos y animales (McLaughlin \& Singh, 1999). El Cd una vez consumido, puede acumularse en el riñón y en el hígado, puede alterar el sistema óseo, produciendo una enfermedad denominada Itai-Itai que fue detectada por la primera vez en Japón (Gupta \& Gupta, 1998).

En suelos agrícolas, las principales fuentes de $\mathrm{Cd}$ provienen de la fertilización fosforada, uso de lodos y residuos industriales (Alloway, 1990; McLaughlin \& Singh, 1999). Con relación a los fertilizantes 
fosforados, la principal materia prima de éstos es la roca fosfórica, constituida principalmente por apatita, que, además de $\mathrm{P}$, contiene $\mathrm{Cd}$ en cantidades que varían entre 8 y $500 \mathrm{mg} \mathrm{kg}^{-1}$ (Laegreid et al., 1999). Por ejemplo, en Chile se ha determinado que la roca fosfórica de Bahía Inglesa contiene alrededor de $6 \mathrm{mg} \mathrm{kg}^{-1}$ de Cd (Valenzuela, 2001). Investigaciones recientes indican que los contenidos de Cd en fertilizantes comercializados en Brasil varían entre 0,6 y 3,5 mg kg-1 (Gabe \& Rodella, 1999; Prochnow et al., 2001). En los Estados Unidos, Australia y Nueva Zelandia existen diversos estudios acerca de la concentración de este elemento en los fertilizantes y el efecto de su acumulación en suelos y plantas (Williams \& David, 1973; Charter et al., 1993; Loganathan et al., 1997; Raven \& Loeppert, 1997). Todos estos estudios indican que la aplicación reiterada de fertilizantes fosforados incrementa las cantidades de Cd en el suelo.

El comportamiento del Cd incorporado al suelo está en función del tipo de reacción química y en los diversos procesos físicos y biológicos que ocurren en el suelo. Las principales reacciones involucradas en las interacciones entre los metales y los componentes del suelo son las de adsorción, precipitación y formación de complejos (Ahumada \& Schalscha, 1995, Helmke, 1999). En suelos incubados con Cd, Barrow (1998) encontró que éste se mantiene en solución a través del tiempo, lo que conserva su efecto tóxico. Laegreid et al. (1999) señalan que la disponibilidad y movilidad del $\mathrm{Cd}$ en el suelo dependen del $\mathrm{pH}$, humedad, materia orgánica, tipo y cantidad de arcilla, además de la cantidad y número de años de aplicación de fertilizantes fosforados. El Cd puede ser absorbido por las plantas como $\mathrm{Cd}^{2+}$ y se encuentra en mayor cantidad en aquellas hortalizas como lechuga, espinacas y apio, que finalmente son consumidas por el hombre (McLaughlin \& Singh, 1999). Recientes estudios en Brasil han demostrado que en plantas de fréjol el Cd se acumula en las raíces, afectando a los rizobios que participan en el proceso de fijación simbiótica de nitrógeno (Matsuda, et al., 2002)

Investigaciones realizadas en Chile señalan que la incorporación de Cd a los suelos se debe principalmente a la actividad mineral e industrial, encontrándose este elemento en mayor cantidad en áreas industrializadas (González \& Ite, 1992). Además se han realizado estudios relacionados con la absorción de Cd en algunos cultivos en suelos contaminados (Ahumada \& Schalscha, 1995), en suelos del orden Andisol no cultivados y prospecciones de los niveles de Cd total en distintas regiones de Chile (González \& Ite, 1992). Sin embargo, no existen antecedentes sobre el efecto de la fertilización fosforada sobre el contenido y acumulación de Cd en los suelos en el tiempo.

El Cd es un elemento no deseable en los fertilizantes y aunque no representa un peligro inmediato debido a su baja concentración (McLaughlin \& Singh, 1999), la industria de fertilizantes está trabajando en bajar su contenido mediante técnicas de eliminación y purificación de los fertilizantes (Isherwood, 1992). Actualmente la estrategia está orientada al uso de los fertilizantes fosforados con bajas concentraciones de este metal. Por esta razón, en lugares como Japón, Australia y algunos países de Europa se han propuesto límites de tolerancia en la concentración de Cd de los fertilizantes fosforados que se comercializan. Cuando el contenido excede el valor limite se debe declarar su concentración en el producto fertilizante. Los países de la Organización para el Desarrollo y Cooperación Económica (OECD) también han propuesto niveles de tolerancia en el contenido de $\mathrm{Cd}$ por kg de $\mathrm{P}$ que aportan, siendo menor de $50 \mathrm{mg} \mathrm{kg}^{-1}$ de $\mathrm{P}$ para Suiza y Finlandia, menor de $100 \mathrm{mg} \mathrm{kg}^{-1}$ de P para Noruega y Suecia, menor de $210 \mathrm{mg} \mathrm{kg}^{-1}$ de $\mathrm{P}$ en el caso de Bélgica y Alemania y hasta 340 mg kg-1 de P en el caso de Japón y Australia (Laegreid et al., 1999).

El objetivo de este estudio fue evaluar el efecto de la fertilización fosforada sobre la concentración de Cd disponible en cuatro tipos de suelos de Chile.

\section{Material y Métodos}

Los suelos seleccionados son de uso agrícola en Chile y pertenecen al orden Alfisol, Inceptisol, Ultisol y Andisol. Los dos primeros se encuentran localizados en la zona central y los otros dos al sur del país. Se tomaron muestras de cada suelo $(0-20 \mathrm{~cm})$ y se les realizó un análisis de laboratorio para determinar $\mathrm{pH}$, materia orgánica, fósforo disponible, bases de intercambio (Ca, Mg, $\mathrm{Na}$ y K), aluminio extractable, micronutrientes $(\mathrm{Cu}, \mathrm{Zn}, \mathrm{Fe}, \mathrm{Mn})$ y Cd disponible. 
ElpH se determinó mediante el método potenciométrico, la medición se realizó en el sobrenadante de una suspensión de una mezcla de suelo y agua, 1:2,5 (v:v). La materia orgánica fue determinada por el procedimiento de Walkey \& Black, que consiste en realizar una combustión húmeda de la materia orgánica del suelo con una mezcla de dicromato de potasio y ácido sulfúrico, luego la solución se tituló con sulfato ferroso y se determinó el carbono orgánico del suelo.

En la determinación del P-Olsen, se realizó una extracción del fósforo disponible con una solución de bicarbonato de sodio a pH 8,5 y luego se agitó la muestra con 180 golpes/minuto, se filtró y el P se determinó por espectrofotometría de absorción molecular (colorimetría). En el filtrado se agregó agua destilada y molibdato-vanadato, se dejó desarrollar el color durante 30 minutos y se leyó en un colorímetro a $882 \mathrm{~nm}$ de longitud de onda, habiendo preparado, leído y graficado previamente una curva de patrón colorimétrica.

El Al extractable, se determinó usando el extractante acetato de amonio a $\mathrm{pH} 4,8$, luego se determinó mediante espectrofotometría de absorción atómica (EAA), usando una llama de óxido nitroso y acetileno.

Para la determinación de las bases de intercambio se realizó una extracción del Ca, Mg, Na y K con una solución de acetato de amonio a pH 7,0, actuando el amonio como catión capaz de desplazar. La determinación de la concentración de bases intercambiables se realizó por EAA, usando una llama de acetileno (Page, 1982).

En el caso de los micronutrientes del suelo como el $\mathrm{Cu}, \mathrm{Fe}, \mathrm{Mn}$ y Zn se realizó una extracción con DTPA y en el extracto se midieron los micronutrientes mediante EAA, usando una llama de acetileno y aire (Lindsay \& Norvell, 1978).

El nivel de Cd disponible en el suelo se determinó empleando EDTA 0,05M a pH 7,0 en una relación suelo:extractante de 1:2 (v:v). Las muestras se agitaron por dos horas y posteriormente se centrifugaron y filtraron. En el filtrado se determinó Cd por EAA, se usó atomtrap con el fin de concentrar mejor la muestra en el suelo y facilitar la lectura, también se utilizó una superlámpara de cadmio, usando una llama de acetileno-aire.

En la medición del contenido de Cd disponible en el tiempo se utilizó la técnica de incubación mediante el método de Barrow \& Shaw (1975). La incubación de suelos es un proceso de simulación de las reacciones químicas, físicas y biológicas que ocurren en el suelo, lo que permite estudiar la evolución de un elemento a través del tiempo. Esta técnica consiste en dar las condiciones de temperatura y humedad que aceleran los diferentes procesos que ocurren en el suelo. A cada suelo se le aplicó el fertilizante fosforado en una dosis de P suficiente para alcanzar el mismo nivel de P-Olsen de $30 \mathrm{mg} \mathrm{kg}^{-1}$, la cual se calculó considerando las distintas capacidades de retención de P, determinada experimentalmente (Valenzuela, 2001). El valor de $30 \mathrm{mg} \mathrm{kg}^{-1}$ fue utilizado, debido a que, según estudios de Rodríguez et al. (2001), es el valor que hace posible la máxima productividad de los cultivos en los suelos estudiados, considerando que no existan otros factores limitantes. El fertilizante aplicado correspondió a un superfosfato triple ingresado a Chile en 1999. Se seleccionó este embarque debido a que en estudios preliminares durante las dos temporadas previas, se observó que éste fue el que presentó la mayor concentración de Cd y además fue el más comercializado en el país durante el año en que se realizó el estudio (Valenzuela, 2001).

$\mathrm{Al}$ inicio de la incubación se llevaron todos los suelos a capacidad de campo y durante la incubación los suelos se mantuvieron en estufa a $25^{\circ} \mathrm{C}$, en bolsas de polietileno. Los tratamientos fueron un testigo y la dosis calculada de P para cada suelo. La incubación se realizó bajo un diseño experimental completamente al azar con medidas repetidas en el tiempo. La unidad experimental fue una bolsa de $250 \mathrm{~g}$ de suelo seco con tres repeticiones. El Cd se analizó a los 1, 2, 7, 14, 21, 36, 49, 63, 77 y 90 días después de la aplicación del fertilizante fosforado al inicio de la incubación.

Los resultados del contenido de cadmio de cada suelo con los dos tratamientos fueron sometidos a un análisis de varianza (ANOVA) de dos vías y las medias fueron separadas por el test de Tukey $(\mathrm{P}<0,05)$.

\section{Resultados y Discusión}

Los resultados de los análisis efectuados a los suelos del estudio se encuentran en el Cuadro 1. En general, los suelos presentaron niveles de P-Olsen inicial en un nivel bajo a muy bajo. El suelo del orden Alfisol, presentó un pH cercano al neutro y bajo contenido de materia orgánica. El suelo del orden Inceptisol, corresponde a un suelo alcalino, con bajo contenido de materia orgánica y destaca por su alta concentración de calcio. El suelo Ultisol, y el Andisol, presentaron $\mathrm{pH}$ ácido.

Se determinó también el Al extractable en que se remueve parcialmente los grupos de $\mathrm{Al}$ estructural de las arcillas y óxidos responsables por la adsorción de P (Rodríguez, 1993). Se ha visto que el Al extractable es un buen indicador de la retención o fijación de $\mathrm{P}$ en el suelo, debido a que se relaciona con la mineralogía de las arcillas. En este caso los valores menores fueron de los suelos Alfisol e 
Inceptisol, valor medio en el Ultisol y valor muy alto en el Andisol (Cuadro 1). Esto último se explica dado que el suelo Andisol contiene principalmente arcillas alófanas que tienen una alta superficie reactiva y una alta proporción del P adsorbido en el pool lábil pasa al pool no lábil (Rodríguez, 1993).

Los suelos difieren en sus características químicas y la concentración de $\mathrm{Cd}$ inicial es estadísticamente distinta para todos los suelos (Cuadro 1). Si se comparan los cuatro suelos, el mayor contenido de Cd inicial se observa en el Inceptisol.

Las cantidades de P y Cd agregadas al suelo vía la fertilización fosforada son presentadas en el Cuadro 2. La mayor dosis de fertilizante corresponde al suelo Andisol debido a su alta capacidad de retención de fósforo. A los 90 dias de incubacíon, en todos los suelos, la cantidad de Cd fue mayor en los tratamientos fertilizados con $\mathrm{P}$ en relación a los testigos sin aplicación de fertilizante $(\mathrm{P}<0,001$ para todos los suelos) (Cuadro 3). Resultados semejantes fueron encontrados por Mulla et al. (1980), Archer \& Hodgson (1987), Holmgren et al. (1993), Roberts et al. (1994) y Mermut et al. (1996).

En las incubaciones no se encontró efecto estadísticamente significativo del tiempo, es decir el Cd liberado el primer día producto de la fertilización, permaneció disponible hasta el último día de medición. De igual forma no se observó interacción significativa entre la dosis y el tiempo de incubación sobre la concentración de Cd disponible en ninguno de los suelos (Cuadro 4).
Respecto al efecto del tiempo de incubación, los resultados concuerdan con lo señalado por Barrow (1998), quién incubó suelos con Cd y encontró que la concentración de este elemento disponible no varió al pasar los días de incubación (Figura 1), y por lo tanto se acumula en el tiempo. Gray et al. (1998) encontraron que el Cd liberado en el suelo aumentó en los primeros cinco días de incubación y luego se mantuvo constante a partir del día 30 hasta los 70 días después de la medición. Sin embargo, otros estudios señalan que la disponibilidad del $\mathrm{Cd}$ disminuyó en el tiempo, dependiendo de las condiciones del suelo como $\mathrm{pH}$, materia orgánica y la cantidad de Cd aplicado (Laegreid et al., 1999).

El suelo Inceptisol tuvo una concentración inicial de $0,105 \mathrm{mg} \mathrm{kg}^{-1}$ y a los 90 días este tratamiento presentó 0,110 mg kg-1 , sin presentar una diferencia estadística significativa para el tiempo de incubación (Cuadro 4). Al aplicar la fertilización fosforada a los 90 días de incubación, el Cd disponible alcanzó niveles de $0,128 \mathrm{mg} \mathrm{kg}^{-1}$ (Figura 1 ). Al final del estudio el aporte neto de $\mathrm{Cd}$ al suelo producto de la fertilización fosforada fue de $0,02 \mathrm{mg} \mathrm{kg}^{-1}$. Dado que se midió la concentración de Cd del fertilizante aplicado se pudo obtener que por cada unidad de $\mathrm{Cd}$ incorporado vía fertilización quedó un 56\% de Cd disponible en el Inceptisol.

En otro estudio realizado por Williams \& David (1973), en un suelo con una concentración de Cd de $0,11 \mathrm{mg} \mathrm{kg}^{-1}$ en el testigo y luego de aplicaciones sucesivas en el tiempo la concentración de Cd

Cuadro 1. Propiedades químicas de los suelos.

\begin{tabular}{|c|c|c|c|c|}
\hline Propiedad & Alfisol & Inceptisol & Ultisol & Andisol \\
\hline $\mathrm{pH}$ & 6,3 & 8,3 & 5,3 & 5,4 \\
\hline $\mathrm{MO}\left(\mathrm{g} \mathrm{kg}^{-1}\right)$ & 13,0 & 17,0 & 80,0 & 195,0 \\
\hline $\left.\mathrm{P}(\mathrm{mg} \mathrm{kg})^{-1}\right)$ & 8,0 & 3,9 & 1,0 & 3,0 \\
\hline $\mathrm{K}\left(\mathrm{mmol}_{\mathrm{c}} \mathrm{kg}^{-1}\right)$ & 3,69 & 3,92 & 2,97 & 10,33 \\
\hline $\mathrm{Na}\left(\mathrm{mmol}_{\mathrm{c}} \mathrm{kg}^{-1}\right)$ & 0,39 & 2,89 & 2,26 & 1,73 \\
\hline $\mathrm{Ca}\left(\mathrm{mmol}_{\mathrm{C}} \mathrm{kg}^{-1}\right)$ & 55,95 & 221,15 & 62,60 & 70,80 \\
\hline $\operatorname{Mg}\left(\mathrm{mmol}_{\mathrm{c}} \mathrm{kg}^{-1}\right)$ & 14,00 & 10,83 & 43,00 & 25,08 \\
\hline $\mathrm{Al}$ extractable $\left(\mathrm{mmol}_{\mathrm{C}} \mathrm{kg}^{-1}\right)$ & 4,22 & 4,44 & 24,77 & 185,88 \\
\hline $\mathrm{Cu}\left(\mathrm{mg} \mathrm{kg}^{-1}\right)$ & 2,10 & 7,60 & 0,25 & 0,85 \\
\hline $\mathrm{Zn}\left(\mathrm{mg} \mathrm{kg}^{-1}\right)$ & 1,28 & 2,61 & 0,16 & 0,82 \\
\hline $\operatorname{Mn}\left(\mathrm{mg} \mathrm{kg}^{-1}\right)$ & 18,4 & 10,0 & 12,80 & 5,80 \\
\hline $\mathrm{Fe}\left(\mathrm{mg} \mathrm{kg}^{-1}\right)$ & 16,7 & 14,3 & 36,00 & 57,90 \\
\hline $\mathrm{Cd}\left(\mathrm{mg} \mathrm{kg}^{-1}\right)^{(1)}$ & $0,0193 \mathrm{c}$ & $0,105 a$ & $0,0097 d$ & $0,027 b$ \\
\hline
\end{tabular}

${ }^{(1)}$ Los valores seguidos de letras distintas difieren estadísticamente por lo test de Tukey al 5\% de probabilidad. 
aumentó a 0,270 mg kg-1. En suelos del orden Alfisol en Nueva Zelandia, la concentración de Cd en los primeros $7 \mathrm{~cm}$ del suelo fue de $0,13 \mathrm{mg} \mathrm{kg}^{-1}$ para el testigo y de $0,16 \mathrm{mg} \mathrm{kg}^{-1}$ para aquellos suelos fertilizados (Roberts et al., 1994). En otros experimentos se muestran concentraciones de $\mathrm{Cd}$ disponible de $0,4 \mathrm{mg} \mathrm{kg}^{-1}$, para un testigo y 3 mg kg-1 después de aplicar $0,011 \mathrm{mg} \mathrm{L}^{-1}$ de solución de nitrato de Cd $\left(\mathrm{CdNO}_{3}\right)$ (Ahumada \& Schalscha, 1995).

Cuadro 2. Cantidad de superfosfato triple (SFT), fósforo (P) y cadmio (Cd) aplicada a los suelos.

\begin{tabular}{lccc}
\hline Tipo de suelo & $\begin{array}{c}\text { SFT } \\
\text { (mg kg }\end{array}$ & $\begin{array}{c}\mathrm{P} \\
\text { suelo) }\end{array}$ & $\begin{array}{c}\mathrm{Cd} \\
\left(\mathrm{mg} \mathrm{kg}^{-1} \text { suelo) }\right.\end{array}$ \\
\hline Inceptisol & 608 & 125 & 0,032 \\
Alfisol & 485 & 100 & 0,025 \\
Ultisol & 1.153 & 240 & 0,061 \\
Andisol & 2.641 & 545 & 0,140 \\
\hline
\end{tabular}

La disponibilidad de un 56\% de Cd en el Inceptisol podría explicarse en parte por el bajo contenido de materia orgánica en estos suelos, ya que de acuerdo a los antecedentes mencionados en la literatura, a menor contenido de materia orgánica mayor disponibilidad del elemento, debido a la menor presencia de partículas con carga negativa (Cowan et al., 1992). En estudios realizados en suelos aluviales de la zona central de Chile, se demostró que el Cd se encuentra enlazado a la materia orgánica y en su ausencia quedaría disponible (Ahumada \& Schalscha, 1995). En este caso la aplicación de fosfatos no influye en la disponibilidad del Cd, ya que las arcillas predominantes presentan baja cantidad de cargas negativas en la superficie de los coloides. En este estudio se encontró que un $44 \%$ de Cd aportado por la fertilización fosforada no queda disponible, lo cual

Cuadro 3. Contenido de cadmio (mg kg-1 de suelo) en el testigo y en los suelos fertilizados a los 90 días de incubación(1).

\begin{tabular}{lcccc}
\hline Tratamiento & Inceptisol & Alfisol & Ultisol & Andisol \\
\hline Testigo & $0,110 \pm 0,008 \mathrm{a}$ & $0,021 \pm 0,002 \mathrm{a}$ & $0,010 \pm 0,0009 \mathrm{a}$ & $0,029 \pm 0,001 \mathrm{a}$ \\
Con fertilización fosforada & $0,128 \pm 0,007 \mathrm{~b}$ & $0,041 \pm 0,005 \mathrm{~b}$ & $0,033 \pm 0,006 \mathrm{~b}$ & $0,081 \pm 0,005 \mathrm{~b}$ \\
\hline
\end{tabular}

${ }^{(1)}$ Medias seguidas de letras distintas por columna difieren entre sí según el test de Tukey al 5\% de probabilidad.

Cuadro 4. Resultados del ANOVA para evaluar el efecto de la aplicación de fertilizante y del tiempo transcurrido, sobre la disponibilidad de cadmio en los cuatro suelos estudiados.

\begin{tabular}{lrrr}
\hline Fuente de variación & $\mathrm{GL}^{(1)}$ & $\mathrm{F}$ & $\mathrm{P}$ \\
\hline & & Inceptisol & $<0,001$ \\
Aplicación de fertilizante & 1 & 124,721 & 0,125 \\
Tiempo & 9 & 1,683 & 0,158 \\
Aplic. fert x tiempo & 9 & 1,569 & \\
Error & 40 & Alfisol & $<0,001$ \\
& 1 & 360,944 & 0,731 \\
Aplicación de fertilizante & 9 & 0,670 & 0,978 \\
Tiempo & 9 & 0,274 & \\
Aplic. fert x tiempo & 40 & Ultisol \\
Error & 1 & 380,083 & $<0,001$ \\
& 9 & 0,708 & 0,698 \\
Aplicación de fertilizante & 9 & 1,107 & 0,380 \\
Tiempo & 40 & & \\
Aplic. fert x tiempo & & Andisol \\
Error & 1 & $2.656,670$ & $<0,001$ \\
& 9 & 0,364 & 0,946 \\
Aplicación de fertilizante & 9 & 0,174 & 0,996 \\
Tiempo & 40 & & \\
Aplic. fert x tiempo & & \\
Error & &
\end{tabular}


se explica por la alta concentración de carbonatos de calcio en estos suelos de $\mathrm{pH}$ alcalino, condición que favorece la precipitación del Cd en forma de carbonatos de Cd (Alloway, 1990). Por otra parte, diversos estudios han demostrado que en la medida que aumenta el pH, disminuye la solubilidad del Cd y la adsorción es mayor (Gray et al., 1998; Evangelou et al., 1999).

El suelo Alfisol, de origen granítico, presentó una baja concentración inicial de Cd (0,02 mg kg-1, Cuadro 1) y al aplicar la fertilización fosforada la concentración de Cd se duplicó con relación al testigo, alcanzando a $0,04 \mathrm{mg} \mathrm{kg}^{-1}$, lo que es estadísticamente significativo (Cuadro 4) para aplicación de fertilizante P (Figura 1). Por cada unidad de Cd aplicado en la fertilización fosforada, un 77\% de Cd quedó disponible. Estos resultados coinciden con lo descrito por Loganathan et al. (1997), quienes estudiaron un suelo del orden Alfisol, en Nueva Zelandia, y encontraron que la concentración de Cd en el testigo fue de $0,02 \mathrm{mg} \mathrm{kg}^{-1}$ y con la aplicación

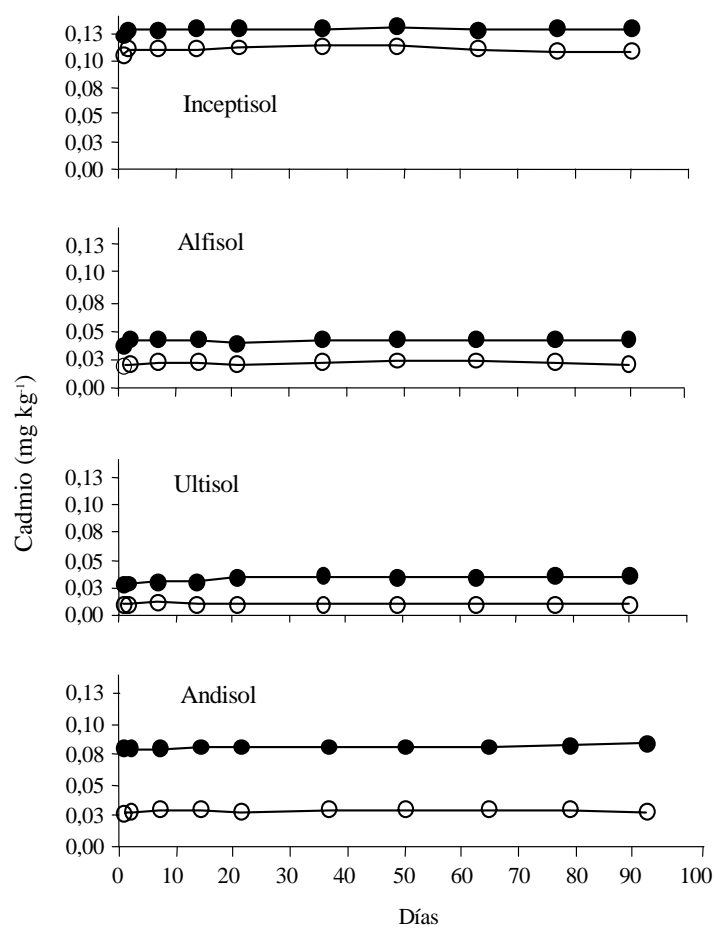

Figura 1. Concentración de cadmio disponible en el tiempo en los suelos con tratamiento testigo (o) y con fertilización fosforada $(\bullet)$. de $60 \mathrm{~kg} \mathrm{ha}^{-1}$ de superfosfato normal, el Cd disponible aumentó a 0,04 $\mathrm{mg} \mathrm{kg}^{-1}$. En un suelo del mismo orden en California, Mulla et al. (1980) encontraron que la concentración de $\mathrm{Cd}$ disponible en el testigo fue mayor que en este caso $\left(0,06 \mathrm{mg} \mathrm{kg}^{-1}\right)$ y que al aplicar una dosis continuada de $175 \mathrm{~kg} \mathrm{ha}^{-1}$ de $\mathrm{P}$ durante 36 años la concentración de Cd disponible en el suelo aumentó a 0,27 $\mathrm{mg} \mathrm{kg}^{-1}$.

En el suelo Ultisol la fertilización fosforada triplicó la concentración de $\mathrm{Cd}$ en el suelo respecto al testigo que presentó $0,01 \mathrm{mg} \mathrm{kg}^{-1}$, efecto que fue estadísticamente significativo (Cuadro 4) para aplicación de fertilizante $\mathrm{P}$ (Figura 1). En este caso se observó que por cada unidad de Cd aplicado proveniente de la fertilización fosforada, un 37\% del Cd quedó disponible. Williams \& David (1973) encontraron que con la aplicación de $230 \mathrm{mg} \mathrm{kg}^{-1}$ de $\mathrm{P}$ la concentración de Cd disponible en el suelo fue de $0,031 \mathrm{mg} \mathrm{kg}^{-1} \mathrm{y}$ el incremento respecto al contenido inicial de Cd producto de la fertilización fosforada fue de $0,025 \mathrm{mg} \mathrm{kg} \mathrm{kg}^{-1}$, cifra muy cercana a los $0,023 \mathrm{mg} \mathrm{kg}^{-1}$ determinados en este estudio con la aplicación de $240 \mathrm{mg} \mathrm{kg}^{-1}$ de fósforo. Aunque este suelo presentó características que favorecen la disponibilidad de Cd en solución (pH ácido), la baja disponibilidad de Cd (37\%) producto de la fertilización fosforada en este tipo de suelo se puede explicar por las características de los suelos del orden Ultisol, que en general presentan alto contenido de óxidos de Fe y Al y también de materia orgánica (Rodríguez, 1993). Esta particular condición ha sido confirmada por Cowan et al. (1992), quienes encontraron que en suelos del orden Ultisol, la disponibilidad de Cd esta controlada por la formación de complejos con la materia orgánica y la presencia de óxidos de hierro.

En el caso del suelo del orden Andisol, el testigo presentó una baja concentración inicial de $\mathrm{Cd}$ disponible $\left(0,027 \mathrm{mg} \mathrm{kg}^{-1}\right.$, Cuadro 1$)$ y al fertilizar con SFT la concentración de este elemento aumentó cuatro veces respecto al valor del testigo, efecto que fue estadísticamente significativo para aplicación de fertilizante $\mathrm{P}$ (Cuadro 4), alcanzando una concentración de $0,08 \mathrm{mg} \mathrm{kg}^{-1}$ al final de la incubación (Figura 1).

El aporte de Cd disponible al suelo producto de la fertilización fosforada en el suelo Andisol fue de $0,06 \mathrm{mg} \mathrm{kg}^{-1}$. Debido a la mayor capacidad de 
retención de P observada en este suelo (Valenzuela, 2001), éste recibió la mayor cantidad de Cd a través del SFT. La principal causa de esto es el hecho de que la disponibilidad de $\mathrm{Cd}$ y de otros elementos como el $\mathrm{P}$ en el suelo está gobernada por distintos factores, tales como el $\mathrm{pH}$. Aún cuando en este suelo las condiciones de $\mathrm{pH}$ ácido favorecen la disponibilidad de Cd en solución, el porcentaje de Cd disponible en base a las unidades de P y Cd agregadas junto al fertilizante fosforado fue sólo de un 37\%. Según Alloway (1990), en suelos ricos en materia orgánica como es este caso (el porcentaje alcanzó a 19\%), la disponibilidad de Cd en solución disminuye por la unión entre el metal y las cargas negativas de la materia orgánica, debido a la formación de quelatos.

Según Ahumada \& Schalscha (1995), en un suelo Andisol a medida que aumenta la cantidad de fosfatos incorporados, suele ocurrir un aumento de la retención de Cd por el incremento de las cargas negativas de la superficie de las arcillas del suelo, producto de la adsorción de fosfatos. Esto crea condiciones electrostáticas favorables para la adsorción de Cd, ya sea en la superficie del suelo o sobre el fosfato adsorbido. Según Krishnarmuti et al. (1999), la aplicación de fosfato puede reducir el contenido de Cd en solución debido a la formación de complejos de fosfatos de cadmio.

Por último, si bien la aplicación del fertilizante fosforado en este estudio no generó concentraciones de Cd en el suelo en niveles críticos, las aplicaciones reiteradas de este tipo de fertilizante pueden ir incrementando paulatinamente los niveles de Cd en el suelo. Esta situación queda de manifiesto al observar que una vez alcanzado el equilibrio, el nivel de Cd disponible aportado por el fertilizante permaneció constante en el tiempo (Figura 1). Los límites críticos propuestos para el nivel de Cd total en el suelo, corresponden a concentraciones entre 3 a 8 mg kg-1 (Alloway, 1990).

\section{Conclusiones}

1. Al agregar dosis agronómicas de fertilizantes fosforados que contienen Cd para la producción de cultivos en los suelos Alfisol, Inceptisol, Ultisol y
Andisol, presentes en distintas regiones de Chile, queda una parte importante de este metal disponible para las plantas.

2. Una vez que el fertilizante fosforado reacciona en el suelo, la cantidad de Cd que queda disponible no disminuye al menos en 90 días de incubación a $25^{\circ} \mathrm{C}$

3. Los suelos Andisoles de Chile necesitan altas dosis de fertilizante para la producción agrícola, ya que presentan una capacidad de retención de P muy alta; así, al utilizar repetidamente fertilizantes fosforados que contienen Cd, también se estarán agregando altas cantidades de este elemento.

\section{Referencias}

AHUMADA, I.; SCHALSCHA, E. Efecto de la sorción de cadmio y cobre en suelos. Agrochimica, Pisa, v. 39, p. 101-110, 1995.

ALLOWAY, B. J. Heavy metals in soils. New York: J. Wiley, 1990. 339 p.

ARCHER, F. C.; HODGSON, I. H. Total and extractable trace element contents of soils in England and Wales. Journal of Soil Science, Aberdeen, v. 38, p. 421-432, 1987.

BARROW, N. J. Effects of time and temperature on the sorption of cadmium, zinc, cobalt and nickel by an soil. Australian Journal of Soil Research, Melbourne, v. 36, p. 941-950, 1998.

BARROW, N. J.; SHAW, T. C. The slow reactions between soils and anions -2 : effects of time and temperature on the decrease in phosphate concentration in soil solution. Soil Science, Baltimore, v. 119, p. 167-177, 1975.

CHARTER, R. A.; TABATAI, M.; SCHAFER, J. W. Metal contents of fertilizers marketed in Iowa. Communications in Soil Science and Plant Analysis, New York, v. 24, n. 9/10, p. 961-972, 1993.

COWAN, C.; ZACHARA, J.; SMITH, S.; RESCH, C. Individual sorbent contributions to cadmium sorption on Ultisols of mixed mineralogy. Soil Science Society of America Journal, Madison, v. 56, p. 1084 -1094, 1992.

EVANGELOU, M.; MARSI, M.; VANDIVIERE, M. Stability of $\mathrm{Ca}^{2+}, \mathrm{Cd}^{2+}, \mathrm{Cu}^{2+}$ illite humic complexes and pH influence. Plant and Soil, Dordrecht, v. 213, p. 63-74, 1999. 
GABE, U.; RODELLA, A. Trace elements in Brazilian agricultural limestones and mineral fertilizers. Communications in Soil Science and Plant Analysis, New York, v. 30, n. 5/6, p. 605-620, 1999.

GONZÁLEZ, S.; ITE, R. Acumulación metálica en suelos del área bajo influencia de las chimeneas industriales de Ventanas (Provincia Valparaíso, V Región). Agricultura Técnica, Santiago, v. 50, n. 2, p. 214-219, 1992.

GRAY, C. W.; McLAREN, R. G.; ROBERTS, A. H.; CONDRON, L. M. Sorption and desorption of cadmium from some New Zealand soils: effect of $\mathrm{pH}$ and contact time. Australian Journal of Soil Research, Melbourne, v. 36, p. 199-216, 1998

GUPTA, U.; GUPTA, S. C. Trace element toxicity relationships to crop production and livestock and human health: implications for management. Communications in Soil Science and Plant Analysis, New York, v. 29, n. 11/14, p. 1491-1522, 1998.

HELMKE, P. A. Chemistry of cadmium in soil solution. In: McLAUGHLiN, M. J.; SINGH, B. R. (Ed.). Cadmium in soils and plants. Dordrecht: Kluwer Academic, 1999. p. 36-64.

HOLMGREN, G.; MEYER, M. W.; CHANEY, R. L.; DANIELS, R. B. Cadmium, lead, zinc, copper, and nickel in agricultural soils of the United States of America. Journal of Environmental Quality, Madison, v. 22, p. 335-348, 1993.

ISHERWOOD, K. F. Phosphate industry and the environment. In: INTERNATIONAL WORKSHOP OF PHOSPHATE FERTILIZER ON THE ENVIRONMENT, 1992, Tampa. Proceedings... Tampa: International Fertilizer Development Center, 1992. p. 115-123.

KRISHNARMUTI, G.; HUANG, P.; KOZAK, L. Sorption and desorption of cadmium form soils: influence of phosphate. Soil Science, Baltimore, v. 164, n. 12, p. 888-898, 1999.

LAEGREID, M.; BOCKMAN, O. C.; KAARSTAD, E. O. Agriculture fertilizers and environment. Wallingford: CAB, 1999. 294 p.

LINDSAY, W. L.; NORVELL, W. A. Development of a DTPA soil test for zinc, iron, manganese and copper. Soil Science Society of America Journal, Madison, v. 42, p. 421-428, 1978.

LOGANATHAN, P.; HEDLEY, M.J.; GREGG, P. E.; CURRIE, L. D. Effect of phosphate fertilizer type on the accumulation and plant availability of cadmium in grassland soils. Nutrient Cycling in Agroecosystem, Dordrecht, v. 47, p. 169-178, 1997

McLAUGHLIN, M. J.; SINGH, B. R. Cadmium in soils and plants: a global perspective. In: McLAUGHLIN, M. J.; SINGH, B. R. (Ed.). Cadmium in soils and plants. Dordrecht: Kluwer Academic, 1999. p. 1-19.

MATSUDA, A.; SOUZA, F. M. de; OSWALDO, J. Tolerância de rizóbios de diferentes procedências ao zinco, cobre e cádmio. Pesquisa Agropecuária Brasileira Brasília, v. 37, n. 3, p. 343-355, mar. 2002.

MERMUT, A. R.; JAIN, J. C.; SONG, L.; KERRICH, R.; KOZAK, L.; JANA, S. Trace element concentrations of selected soils and fertilizers in Saskatchewan, Canada. Journal of Environmental Quality, Madison, v. 25, p. $845-853,1996$

MULLA, D.; PAGE, A.; GANJE, T. Cadmium accumulations and bioavailability from long term phosphorus fertilization. Journal of Environmental Quality, Madison, v. 9, n. 3, p. 408-412, 1980.

PAGE, A. L. Methods of soil analysis: chemical and microbiological properties. $2^{\text {nd }}$ ed. Madison: American Society of Agronomy/Soil Science Society of America, 1982. pt. 2. (Agronomy Series, 9).

PROCHNOW, L. I.; PLESE, L. M.; ABREU, M. F. Bioavailibity of cadmium contained in single superphosphates produced from different Brazilian raw materials. Communications in Soil Science and Plant Analysis, New York, v. 32, n. 1/2, p. 283-294, 2001.

RAVEN, K. P.; LOEPPERT, R. H. Trace element composition of fertilizers and soil amendments. Journal of Environmental Quality, Madison, v. 26, p. 551-557, 1997. ROBERTS, A. H.; LONGHURTS, R. D.; BROWN, M. W. Cadmium status of soil, plants, and grazing animals in New Zealand. New Zealand Journal of Agricultural Research, Wellington, v. 37, p. 119-129, 1994.

RODRÍGUEZ, J. La fertilización de los cultivos: un método racional. Santiago: Pontificia Universidad Católica de Chile, 1993. 291 p.

RODRÍGUEZ, J.; PINOCHET, D.; MATUS, F. La fertilización de los cultivos. Santiago: LOM, 2001. 117 p.

VALENZUELA, A. Determinación de cadmio en fertilizantes fosforados y su efecto en cuatro tipos de suelos de Chile. $80 \mathrm{f}$. Tesis (Magister en Ciencias Agropecuarias) - Pontificia Universidad Católica de Chile, Santiago, 2001.

WILLIAMS, C. H.; DAVID, D. J. The effect of superphosphate on the cadmium content of soils and plants. Australian Journal Soil Research, Melbourne, v. 11, p. 43-56, 1973. 